\title{
La responsabilidad social en la formación de los abogados
}

\author{
LORENZO ZOLEZZI IBÁRCENA*
}

\begin{abstract}
SUMARIO: I. EL PARADIGMA UNIVERSITARIO CLÁSICO.- II. EL NUEVO PARADIGMA.- III. DE LA SOLIDARIDAD AL INDIVIDUALISMO Y NUEVAMENTE A LA SOLIDARIDAD.- IV. LA GESTIÓN INTERNA.- V. LA DOCENCIA.- VI. LOS PROFESORES.- VII. LA PROYECCIÓN SOCIAL.- VIII. A MODO DE CONCLUSIÓN.
\end{abstract}

\section{EL PARADIGMA UNIVERSITARIO CLÁSICO}

Tradicionalmente se ha asignado a la universidad tres grandes tareas: (a) la creación del conocimiento, a través de las investigaciones de los profesores, de sus reflexiones y, en general, del cultivo de su disciplina; (b) la transmisión del conocimiento, ya sea del corpus de conocimientos existentes en una materia o de estos y de aquellos generados por las investigaciones y aportaciones científicas de sus propios profesores; (c) la proyección social y la extensión universitaria, en la medida en que la universidad —en particular la universidad pública — es financiada por el erario nacional y en que a ella se le asignan recursos que podrían tener otro destino, como la educación primaria y secundaria o la salud.

En retribución, la universidad se convierte en el centro de la producción de conocimientos y de la formación de profesionales, cuyo impacto en la vida nacional debe ser mucho mayor que en el caso de que los fondos destinados a ella se hubieran invertido en otra cosa. Dentro de la misma lógica, la universidad debe proyectarse hacia la comunidad extendiendo el saber a quienes no forman parte de la comunidad universitaria a través de una serie de mecanismos, como la prestación de servicios a los sectores público y privado; la investigación aplicada, que apunta a solucionar problemas específicos de la comunidad; y la realización de tareas concretas de servicio, como por ejemplo campañas de vacunación, consultorios jurídicos o alfabetización.

\section{EL NUEVO PARADIGMA}

Una nueva manera de concebir la empresa, en su relación con el entorno más cercano y con el país, ha dado lugar al tema de la responsabilidad social empresarial, que después de algunos años de desarrollos teóricos y prácticos ha sido recibido en la universidad como la responsabilidad social universitaria o RSU. 
En realidad, se trata de un nuevo paradigma que vuelve a pensar la universidad a partir de conceptos que la atraviesan transversalmente, y que en consecuencia afecta su propia gestión interna, su manera de concebir y hacer ciencia, su manera de enseñar y su manera de insertarse en la comunidad que le es más cercana y en el país.

\section{DE LA SOLIDARIDAD AL INDIVIDUALISMO Y NUEVAMENTE A LA SOLIDARIDAD}

Cuando yo ingresé a la universidad en 1960, la gran agenda mundial y, por tanto, nacional, era el desarrollo. Se pensaba con optimismo que los países llamados «subdesarrollados» podían dejar de serlo a través de políticas públicas y de un compromiso de todas las instituciones con esa quimera. La universidad, por cierto, tomó dicha bandera y asumió el compromiso de distintas formas: algunos sectores de estudiantes y profesores se dedicaron a profundizar en aspectos teóricos que venían del marxismo, de la experiencia de la Unión Soviética y del reciente triunfo de la revolución cubana en 1959: su trabajo fue ideológico y gestó una serie de grupos políticos que fueron bastante activos especialmente en las universidades públicas. Otros sectores, más radicalizados, decidieron pasar a la acción: tal fue el caso del poeta Javier Heraud, quien murió a los 21 años en el río Madre de Dios, el 15 de mayo de 1963, empeñado en un proyecto revolucionario que no tenía posibilidad alguna de éxito; el levantamiento de Luis de la Puente Uceda, en 1964, fue también desarticulado y exterminado.

Pero había otra línea de acción basada en la ayuda social, en el compromiso teórico y práctico con la lucha contra la pobreza. Este dejaba al Estado el cambio de estructuras que, asumiéndose que ocurriera de manera eficiente y no traumática, debía conjugarse con la acción solidaria y producir una serie de cambios incrementales que, a la larga, sacarían al país del estancamiento. Sobre esta línea se inscribió la Alianza para el Progreso, un programa de ayuda económica y social impulsada por los Estados Unidos; el voluntariado ejercido por los cuerpos de paz, también de origen norteamericano; y aquel interesantísimo proyecto ideado por el presidente Fernando Belaúnde Terry llamado Cooperación Popular, que tuvo un capítulo denominado Cooperación Popular Universitaria.

Estudiantes de todas las especialidades y de todas las universidades del país se movilizaron masivamente hacia el campo para trabajar con las comunidades en obras de infraestructura, en control de plagas, en capacitación sanitaria, en alfabetización, en estudio de títulos de propiedad, en la formación de cooperativas, etcétera. Durante varios años, miles de estudiantes pasaron por estas experiencias y se generó una verdadera corriente de solidaridad universitaria con los pobres, con los desposeídos, con los pueblos olvidados. 
La Pontificia Universidad Católica del Perú (en lo que sigue me referiré a ella con su nombre completo o simplemente con sus siglas: PUCP) organizó su propio servicio de voluntariado, denominado Campamento de Trabajo, a través de la Federación de Estudiantes y con el apoyo oficial del Rectorado. Colocó a cincuenta estudiantes de diversas carreras profesionales en el pueblo de Nuevo Imperial, en la provincia de Cañete, donde trabajaron hombro a hombro con los pobladores durante un mes.

Este espíritu de solidaridad y de compromiso con el desarrollo del país penetró profundamente en la Facultad de Derecho de la PUCP. Se firmó un convenio con la Universidad de Wisconsin, a la sazón una de las universidades más progresistas de los Estados Unidos y cuna de los estudios jurídicos enfocados en su relación con la sociedad. El objetivo era enviar profesores durante períodos largos y cortos a dicha casa de estudios para familiarizarlos con el método de enseñanza socrático, basado en la lectura previa, por parte de los alumnos, de un material preparado y dado a leer por el profesor, lo cual hacía de la clase un espacio de diálogo e intercambio.

Los grandes maestros de esa corriente que se denominó Law and Society, «Derecho y sociedad», eran profesores en Wisconsin: Lawrence Friedman, Stewart Macaulay, Robert Seidman, Jack Ladinsky y otros. Ello contribuyó también a que los profesores peruanos participantes de esa experiencia dotaran a sus cursos de un enfoque dirigido a contrastar el Derecho con la realidad.

Pero la Católica no fue la única universidad Latinoamericana en embarcarse en proyectos de este tipo. Lo hicieron también varias universidades chilenas, que se asociaron con Stanford. En 1971 tuvo lugar en Valparaíso la Primera Conferencia sobre la Enseñanza del Derecho y el Desarrollo, organizada por el Consejo de Decanos de las Facultades de Derecho de Chile y el Instituto de Docencia e Investigación Jurídicas de Chile. La segunda conferencia se llevó a cabo en Lima, en 1973, y fue organizada por la Pontificia Universidad Católica del Perú. Por esa época una organización no gubernamental norteamericana, el International Legal Center, constituyó dos comités: el primero debía estudiar el tema de la educación legal, estaba conformado por dieciséis destacados juristas del mundo entero y fue presidida por el entonces decano de la Católica, el doctor Jorge Avendaño Valdez. El segundo comité tenía el encargo de estudiar el futuro de la investigación en Derecho y desarrollo y estuvo integrado por diecisiete juristas, de los cuales el único latinoamericano fue el que ahora escribe estas líneas. Ambos comités produjeron sendos informes; existió una versión en castellano del Informe sobre la Investigación en Derecho y Desarrollo․ Por aquellos años se constituyó el Consejo Latinoamericano de Derecho y Desarrollo, que 
organizó las siguientes conferencias sobre la enseñanza del Derecho y el desarrollo en Costa Rica, Buenos Aires y Caracas.

Todo este vasto movimiento dejó un sello imborrable en algunas de las facultades de Derecho que se involucraron a fondo, como es el caso de la Facultad de Derecho de la Universidad Católica del Perú, pero en otros casos los temas que interesaban al movimiento no fueron bien vistos, como ocurrió en el Chile de Pinochet. Y, en general, la década del ochenta y más específicamente la del noventa no fueron propicias para el mantenimiento del espíritu solidario, sino más bien para que se perfilara entre el estudiantado una actitud más bien indiferente ante lo social y centrada específicamente en el interés individual por terminar los estudios, graduarse y destacar, fomentado todo esto por el incremento del número de facultades de Derecho, la figura del bachillerato automático y la aparición de los cursos especiales de titulación. Todo lo anterior produjo una gran cantidad de abogados y, consecuentemente, una fuerte competencia en el mercado laboral.

Sostengo que el paradigma de la responsabilidad social universitaria supone una vuelta al compromiso con la solidaridad a partir de la siguiente definición:

La Responsabilidad Social Universitaria es una política de gestión de la calidad ética de la Universidad que busca alinear sus cuatro procesos (gestión, docencia, investigación, extensión) con la misión universitaria, sus valores y compromiso social, mediante el logro de la congruencia institucional, la transparencia y la participación dialógica de toda la comunidad universitaria (autoridades, estudiantes, docentes, administrativos) con los múltiples actores sociales interesados en el buen desempeño universitario y necesitados de él, para la transformación efectiva de la sociedad hacia la solución de sus problemas de exclusión, inequidad, y sostenibilidad. ${ }^{2}$

La PUCP ha precisado su misión, visión y valores de la siguiente forma:

La Pontificia Universidad Católica del Perú es una comunidad académica inspirada en principios éticos y valores católicos, creadora y difusora de cultura, saber y conocimiento, promotora del cambio, dedicada a la formación integral de la persona, para que ella haga del estudio un instrumento de su propia realización y se capacite para asumir y resolver problemas fundamentales inherentes al ser humano y a la sociedad.

Nuestra visión es liderar la formación universitaria en el país y ser reconocida nacional e internacionalmente como espacio promotor y generador de desarrollo. 
Los valores constituyen la base de nuestra cultura organizacional y significan elementos esenciales que forjan la identidad de nuestra universidad, le otorgan singularidad y afirman su presencia social.

Ellos son:

LA RESPONSABILIDAD SOCIAL

Búsqueda de la verdad.

EN LA FORMA-

Respeto por la dignidad de la persona.

Pluralismo.

Responsabilidad social y compromiso con el desarrollo.

Honestidad.

Solidaridad.

Justicia. $^{3}$

\section{LA GESTIÓN INTERNA}

Una de las novedades de la responsabilidad social universitaria es el rol que le asigna a la manera en que la universidad se organiza a sí misma. Sostiene que los alumnos no aprenden solamente en el aula, sino también y muy destacadamente de su vivencia institucional. Los estudiantes permanecen en la universidad varios años de su vida (podrían ser hasta diez, si consideramos la maestría y el doctorado), de los 17 a los 25 ó 27 (los mejores años, dirían los poetas). Pero, además, pasan en la universidad prácticamente todo el día. Entonces, el estudiante se socializa en la universidad: aprende a ser solidario, a ser democrático, a ser participativo, a no discriminar. Y esto lo aprende mirando a su alrededor: constatando que en la universidad hay alternancia en el gobierno, hay participación estudiantil en la elección de autoridades y en la gestión de las unidades académicas, hay democracia, hay un comportamiento ético generalizado en sus profesores y en los trabajadores administrativos, hay preocupación por el impacto ambiental que puede generar la vida del campus y que existen políticas y prácticas para atenuar tal impacto.

Muy importante en este rubro es la imagen que la universidad proyecta de sí misma cuando realiza campañas para atraer postulantes o simplemente cuando se hace presente en los medios de comunicación para hacer saber lo que hace o lo que logra. No ayudan a fomentar el espíritu de solidaridad que está en la base de la responsabilidad social universitaria campañas publicitarias que hemos visto en Lima hace algunos años de universidades que le dicen al postulante «haremos de ti un león» o «haremos de ti un tiburón». Son frases que transmiten la idea de que la vida profesional es una selva o un peligroso mar donde solo podrá imperar un auténtico depredador, y que la universidad asume el rol de formar esos depredadores. Se han visto también campañas recientes que dicen algo así como «desde que ingresas ya estás trabajando» 0 «sin práctica no 
hay verdadero aprendizaje», mensajes que menosprecian todo el aspecto formativo y de reflexión que es parte de la esencia de la universidad.

Afortunadamente, en nuestra universidad en general y en la Facultad de Derecho en particular se registran condiciones de una muy razonable extensión de la responsabilidad social universitaria en la gestión. Una enumeración parcial de algunos aspectos específicos es la siguiente:

1. Los estudiantes deben participar —y participan — en la elección de sus autoridades.

2. Los estudiantes deben participar - y participan — en el gobierno de la Facultad.

3. La Facultad debe dar facilidades a los estudiantes - y lo hacepara que organicen revistas y asociaciones.

4. La Facultad debe propiciar con cierta periodicidad la participación de los estudiantes en la realización de un diagnóstico integral de ella misma y en la propuesta de medidas para mejorar o modificar algunos aspectos. En la actualidad, por ejemplo, nos encontramos en las etapas preliminares de un claustro pleno que tendrá lugar hacia fin de año y en el cual intervienen profesores, estudiantes y exalumnos.

\section{LA DOCENCIA}

Hablar de docencia supone hablar del proceso de enseñanza-aprendizaje. Hace referencia al perfil de egresado que se busca, al plan de estudios, al perfil del profesor y a la metodología de la enseñanza.

En cuanto a la metodología de la enseñanza, el pensamiento de la responsabilidad social universitaria incide en una serie de aspectos sobre los cuales el movimiento de la reforma de los estudios de Derecho en los años sesenta del siglo XX trabajó intensamente. En algunas facultades de Derecho, como en la de la Pontificia Universidad Católica del Perú, las ideas rectoras permanecen y existe un esfuerzo sostenido por mantenerlas e implementarlas. Es importante, sin embargo, mencionar algunas de ellas:

1. Los estudiantes deben llegar a la Facultad de Derecho con una formación humanista amplia, con adecuados hábitos de lectura, con un buen nivel de comprensión de esta, con conocimientos sobre metodología de la investigación, con experiencia en la redacción de alguna breve monografía. Nosotros confiamos en que esa formación la obtienen en Estudios Generales Letras. La PUCP es la única universidad que posee las facultades de Estudios Generales Letras y Estudios Generales Ciencias, en las cuales los estudiantes reciben una formación básica durante dos años antes de pasar a las respectivas facultades profesionales. 
2. El currículo debe ser flexible, con cursos obligatorios y cursos electivos. Entre los cursos obligatorios deben estar los formativos y los que permiten al alumno asomarse hacia otras disciplinas. Son el tipo de cursos que reclama la responsabilidad social universitaria. En la Facultad de Derecho de la PUCP cabe mencionar Sociología del Derecho, Metodología de la Investigación Jurídica (dos cursos), Ética y Responsabilidad Profesional. En los cursos electivos es donde el alumno va delineando un cierto grado de especialidad profesional.

3. Sobre la misma línea de no formar profesionales encerrados en sus propias disciplinas, la Facultad debe permitir al alumno llevar un número determinado de créditos en otras facultades. En la Facultad de Derecho de la PUCP se permite llevar hasta nueve créditos en cursos llamados «de libre disposición», que se convalidan hasta por tres electivos innominados: electivos 1,2 y 3 . Pero también se permite llevar cursos en otras facultades y convalidarlos por cursos específicos.

4. En cuanto a metodología de la enseñanza, las ideas matrices permanecen: (i) es deseable que el profesor elabore un texto de materiales de enseñanza, que contenga doctrina, jurisprudencia, casos, problemas, etcétera, y que el alumno debe ir leyendo paulatinamente a lo largo del semestre; (ii) la clase se concibe como un espacio de diálogo y debate a partir de lo leído, y corresponde al profesor llevar el hilo conductor hacia las proposiciones que el alumno debe aprender; (iii) la discusión en clase debe estar siempre anclada en la realidad: el Derecho, al fin de cuentas, es una manera de organizar la realidad y de solucionar los conflictos; (iv) hay cursos que por su naturaleza poseen un material de lectura básicamente doctrinario, en cuyo caso es deseable que el profesor fomente la participación de los estudiantes para esclarecer al máximo los conceptos fundamentales y que procure poner dichos conceptos en relación con ejemplos tomados de la realidad o de su propia experiencia profesional; (v) la clase magistral se sigue empleando pero se encuentra atenuada por una búsqueda permanente de participación del alumno para proponer ejemplos o para hacer comparaciones con temas o conceptos de otros cursos o de otras disciplinas.

Existen, por cierto, nuevas técnicas que se aplican en el proceso enseñanza aprendizaje, pero que todavía no han llegado al Derecho. Lo más lejos a lo que han llegado nuestros profesores es al uso de Power Point, pero más como una pizarra electrónica donde han colocado lo que normalmente forma parte de una clase magistral y que van leyendo a lo largo de clase. Representa una manera de enseñar que, personalmente, me parece contraproducente: primero, porque priva al alumno de la capacidad de seguir y entender un discurso oral, elemento básico

LA RESPONSABILIDAD SOCIAL

EN LA FORMACIÓN DE LOS ABOGADOS 
en la formación de un abogado; segundo, porque los alumnos solicitan y obtienen del profesor las láminas utilizadas y estudian a partir de ellas, lo cual los aleja de la consulta bibliográfica y de la lectura de textos complejos.

Cuando hago referencia a nuevas técnicas, me refiero a los cursos interdisciplinarios, dictados conjuntamente por profesores de diferentes departamentos para alumnos de distintas especialidades, que giran alrededor de problemas o de proyectos; a las clínicas jurídicas; a las tecnologías de la información y la comunicación; y al empleo de redes sociales. Todo esto se inscribe dentro de la tendencia actual que existe en la educación superior moderna de propender a la formación por competencias.

El perfil del egresado universitario que postula la responsabilidad social universitaria es el siguiente:

Un estudiante preocupado por las injusticias a su alrededor y con voluntad de comprometerse en acciones concretas. Un estudiante que haya podido desarrollar su propia capacidad solidaria en acciones de voluntariado conducidas desde la Universidad.

Un estudiante informado, capaz de contextualizar su saber especializado en vista a la solución de los problemas cruciales de su sociedad.

Un estudiante capaz de escuchar, intercambiar y entrar en empatía con el otro, es decir que haya podido beneficiar de experiencias sociales formativas a nivel emocional. Un estudiante formado a la ética del diálogo.

Un estudiante promotor de democracia y participación, que sabe ser ciudadano, es decir que «sabe gobernar y ser gobernado» como decía Aristóteles».4

\section{LOS PROFESORES}

La filosofía de la responsabilidad social universitaria no se ha puesto en el caso de una facultad de Derecho, en la cual predominan los profesores por horas y que no son investigadores sino abogados en ejercicio que dedican a la docencia algunas horas a la semana, de preferencia en las primeras horas de la mañana o las últimas de la mañana o la tarde para no interferir con su ejercicio profesional. La Facultad de Derecho de la PUCP cuenta actualmente con trescientos docentes de los cuales unos treinta son de tiempo completo, es decir el 10\%. Es un porcentaje bajo, si se lo compara con Ciencias e Ingeniería, Humanidades o Ciencias Sociales. Varios de estos treinta profesores a tiempo completo ocupan cargos, ya sea en el área de Derecho o en la universidad, con lo cual el número de profesores dedicados exclusivamente a la enseñanza y a la investigación disminuye considerablemente. Sin embargo, algunas proposiciones generales se pueden enunciar: 
1. La Facultad de Derecho debe procurar tener un determinado número de profesores a tiempo completo despojados de tareas administrativas, de manera que puedan embarcarse en proyectos de investigación.

2. Sobre la investigación, Vallaeys nos dirá:

La Universidad orienta la producción del saber y las tecnologías, influye en la definición de lo que se llama socialmente «Verdad, Ciencia, Racionalidad, Legitimidad, Utilidad, Enseñanza, etc.» Incentiva (o no) la fragmentación y separación de los saberes al participar en la delimitación de los ámbitos de cada especialidad. Articula la relación entre tecnociencia y sociedad, posibilitando (o no) el control y la apropiación social de la ciencia. Genera actitudes como elitismo científico, «expertocracia» o al contrario promueve la democratización de la ciencia. Influye finalmente sobre la definición y selección de los problemas de la agenda científica. (iQué tipo de conocimientos producimos, para qué y para quiénes? ¿Qué conocimientos debemos producir y cómo debemos difundirlos para atender las carencias cognitivas que perjudican el Desarrollo social sostenible en el país?) $)^{5}$.

El largo párrafo que precede es en verdad una agenda para organizar el quehacer cognitivo y epistemológico de la universidad. En Derecho, sin embargo, y en el estado actual de la producción en investigación, prefiero una aproximación más modesta: los proyectos de investigación deben ser relevantes para la sociedad o tener, por lo menos, un anclaje en la realidad. Pero no excluyo, de ninguna manera, los grandes esfuerzos doctrinarios, los trabajos de filosofía y lógica jurídicas y todos aquellos que buscan esclarecer la relación entre el Derecho y diversas manifestaciones de la cultura, pues en última instancia la relevancia de la investigación será mayor cuanto más nos permita penetrar en la naturaleza humana y en las vicisitudes de su vida en sociedad.

\section{LA PROYECCIÓN SOCIAL}

La responsabilidad social universitaria califica de «filantropía» los proyectos tradicionales de proyección social y llega, inclusive, a mirarlos con sospecha en la medida en que podrían estar sirviendo de «fachada» a un currículo oculto, centrado en la promoción de un profesional individualista ${ }^{6}$.

Los entiende y los promueve en la medida en que estén articulados en la cosmovisión universitaria. Prefiere los proyectos interdisciplinarios, pertinentes a los problemas del desarrollo y articulados con la educación formal. Es decir, proyectos en los que el estudiante aprende haciendo, 
dentro de cursos oficiales que otorgan créditos. En esta línea se destaca un curso como PROSODE (Proyección Social en Derecho), parte del plan de estudios de la Facultad de Derecho de la PUCP, que, según Vallaeys, «constituye un perfecto ejemplo de integración entre la docencia y el voluntariado con fines sociales, que puede servir de modelo para diseñar nuevas metodologías de enseñanza»?

En este terreno adquiere particular importancia la formación continua. Se entiende por formación continua:

[...] el proceso de formación permanente e integral que acompaña a la persona durante toda su vida, enriqueciendo las diferentes áreas o dimensiones que integran la complejidad del ser humano: personal, moral, laboral, social, cultural, etc. Este proceso formativo, que la Universidad asume como función primordial entre las tareas que debe cumplir en la sociedad, no otorga grado académico de magíster o doctor, ni título profesional de licenciado, ni ninguna certificación equivalente a éstas.

Este proceso comprende dos tipos de formación continua:

a. De carácter académico y profesional: son aquellas actividades de formación continua que buscan mejorar el conocimiento y las competencias profesionales de la persona en el mundo laboral en el que está inserta. Es una formación continua para el trabajo.

b. De carácter personal: son aquellas actividades de formación continua que buscan el enriquecimiento y la mejora de la calidad de vida de la persona. Es una formación continua para la vidas.

Una facultad de Derecho tiene un campo vastísimo para desarrollar formación continua. En primer lugar, las leyes permanentemente experimenta transformaciones o aparecen nuevas regulaciones jurídicas. Esto genera en sus egresados una necesidad constante de actualización o reciclaje. En segundo lugar, no hay problema social que no posea o no exija una norma jurídica que lo regule, así como existen normas jurídicas que deben ser conocidas por todas la población, para lograrse la eficacia que buscaron los legisladores al aprobarla. Ello genera una necesidad social que solo una facultad de Derecho puede afrontar.

De este modo, nuestra Facultad de Derecho puede cumplir una tarea fundamental en la responsabilidad social universitaria. Primero, en el frente de sus egresados, al hacerlos volver periódicamente a las aulas para adiestrarlos en las nuevas normas jurídicas y para hacerlos reflexionar sobre la relación entre las modificaciones normativas y los cambios sociales. Segundo, en el frente de la sociedad en general, al asumir la tarea de formar buenos ciudadanos, conocedores y respetuosos de la ley, o críticos de ser el caso. Esta es una tarea que impone la responsabilidad social universitaria, consistente en la proyección de la universidad hacia 
la comunidad en búsqueda de la promoción de ciudadanos responsables, imbuidos en valores y comprometidos con el progreso social.

\section{A MODO DE CONCLUSIÓN}

Según el profesor Gorki Gonzales ${ }^{9}$, existen actualmente en el Perú 97 mil abogados y 98 facultades de Derecho (20 públicas, 44 privadas y 34 filiales autorizadas), las cuales «producen» miles de abogados al año. Los efectos de esta sobreproducción de abogados son previsibles: un gran número de ellos, sin una adecuada base ética y con muy pocos conocimientos de los fundamentos del Derecho, incrementará las huestes de abogados ambulantes y de los estratos más bajos de la administración pública, del Poder Judicial y del Ministerio Público, con el peligro de que, al poco tiempo, sean abogados, jueces o fiscales a cargo del sistema de administración de justicia.

Frente a este fenómeno, las facultades de Derecho de las universidades privadas que acogen mayormente a los estudiantes de los sectores sociales altos tienden de manera instintiva a replegarse hacia un modelo que privilegia la formación para la actividad empresarial y el mercado, y donde se corre el peligro de que los conocimientos sobre el ser humano, la sociedad, la desigualdad, la exclusión y el atraso puedan considerarse superfluos.

Por todo esto, resulta imperioso que las facultades de Derecho definan su misión con el mayor cuidado y que traten de alinear sus cuatro procesos (gestión, docencia, investigación y extensión) con los postulados de la responsabilidad social universitaria. 